PROCEEDINGS OF THE

AMERICAN MATHEMATICAL SOCIETY

Volume 133, Number 3, Pages 865-874

S 0002-9939(04)07671-3

Article electronically published on September 29, 2004

\title{
EXPONENTIAL DECAY OF CORRELATIONS FOR SURFACE SEMI-FLOWS WITHOUT FINITE MARKOV PARTITIONS
}

\author{
VIVIANE BALADI AND BRIGITTE VALLÉE
}

(Communicated by Michael Handel)

\begin{abstract}
We extend Dolgopyat's bounds on iterated transfer operators to suspensions of interval maps with infinitely many intervals of monotonicity.
\end{abstract}

\section{Statement of Results}

Let $0<c_{1}<\ldots<c_{m}<c_{m+1}<\ldots<1$ be a finite or countable partition of $I=[0,1]$ into subintervals, and let $T: I \rightarrow I$ be so that $\left.T\right|_{\left(c_{m}, c_{m+1}\right)}$ is $C^{2}$ and extends to a homeomorphism from $\left[c_{m}, c_{m+1}\right]$ to $I$. We assume that $T$ is piecewise uniformly expanding: there exist $C \geq 1$ and $\hat{\rho}<1$ so that $|h(x)-h(y)| \leq C \hat{\rho}^{n}|x-y|$ for every inverse branch $h$ of $T^{n}$ and all $n$. Let $\mathcal{H}$ be the set of inverse branches $h: I \rightarrow\left[c_{m}, c_{m+1}\right]$ of $T$. We suppose (Renyi's condition) that there is a $K>0$ so that every $h \in \mathcal{H}$ satisfies $\left|h^{\prime \prime}\right| \leq K\left|h^{\prime}\right|$. Let $r: I \rightarrow \mathbb{R}_{+}$be so that $\left.r\right|_{\left(c_{m}, c_{m+1}\right)}$ is $C^{1}$, and $\inf r>0$. Assume that there is $\sigma_{0}<0$ so that $\sum_{h \in \mathcal{H}} \sup \exp (-\sigma(r \circ h))\left|h^{\prime}\right|<\infty$ for all $\sigma>\sigma_{0}$, and that $\left|r^{\prime} \circ h\right| \cdot\left|h^{\prime}\right| \leq K$ for all $h \in \mathcal{H}$. For $n \geq 1$, write $r^{(n)}(x)=\sum_{k=0}^{n-1} r\left(T^{k}\right)(x)$.

We study the transfer operators, indexed by $s=\sigma+i t$,

$$
L_{s} f(x)=\sum_{T(y)=x} e^{-s r(y)} \frac{f(y)}{\left|T^{\prime}(y)\right|}=\sum_{h \in \mathcal{H}} e^{-s r(h(x))}\left|h^{\prime}(x)\right| \cdot(f \circ h)(x),
$$

acting on $C^{1}(I)$, with norm $\|f\|=\sup |f|+\sup \left|f^{\prime}\right|$. Note that the $L_{s}$ are the transfer operators associated to the (Fourier transform of the correlation function for the) absolutely continuous invariant probability measure of the suspension semiflow defined by $\phi^{t}(x, s)=(x, s+t)$ on the branched surface $\left\{(x, s) \in I \times \mathbb{R}_{+} \mid s \leq\right.$ $r(x)\} / \sim$, with $(x, r(x)) \sim(T(x), 0)$. See e.g. 5].

Finally, the following assumption is a translation of Dolgopyat's "uniform nonintegrability of foliations" condition (see [1, 5, 6] for formulations closer to ours): we say that the pair $(T, r)$ satisfies $U N I$ if there exist $D>0$ and $n_{0} \geq 1$ such that, for every integer $n \geq n_{0} \geq 1$, there exist two elements $h, k$ of the set $\mathcal{H}_{n}$ of inverse branches of $T^{n}$ so that the function on $I$ defined by $\psi_{h, k}(x):=$ $r^{(n)}(h(x))-r^{(n)}(k(x))$ satisfies $\inf \left|\psi_{h, k}^{\prime}\right| \geq D$. (See also Remark 2.5])

Received by the editors November 23, 2003.

2000 Mathematics Subject Classification. Primary 37C30, 37D50, 37E35.

This work was supported in part by two CNRS MATHSTIC grants. We thank A. Hachemi for a careful reading of a previous version of the paper.

(C)2004 American Mathematical Society Reverts to public domain 28 years from publication 
To state our main result, we use the equivalent norms $\|f\|_{1, t}=\sup |f|+\frac{\sup \left|f^{\prime}\right|}{|t|}$, for $|t| \geq \epsilon_{0}>0$, on $C^{1}(I)$.

Theorem 1.1. Let $T$ and $r$ satisfy the assumptions above (in particular, UNI for $D)$. Then there is $A \geq n_{0}$ and $\gamma<1$ so that for all $\sigma$ close enough to 0 , all $|t| \geq \max (2 \pi / D, 4)$, and all $n \geq A \log |t|$, we have $\left\|L_{s}^{n}\right\|_{1, t} \leq \gamma^{n}$.

Theorem 1.1 was proved by Dolgopyat [3] when $\mathcal{H}$ is finite. In [2], we considered the special case when $T(x)=\{1 / x\}$ (or analogues of the Gauss map) and $r=$ $\log \left|T^{\prime}\right|$, working with a different version of $U N I$, adapted to "algebraic" situations. Note that the present $U N I$ assumption also holds in the setting of [2]: if $h \in \mathcal{H}_{n}$ is a linear fraction $(a x+b) /(c x+d)$, then $h^{\prime \prime}(x) / h^{\prime}(x)=-2 c /(c x+d)$ so that $\left|\psi_{h, \hat{h}}^{\prime}(x)\right|=|2[c /(c x+d)-\hat{c} /(\hat{c} x+\hat{d})]|$. Write $\mathcal{F}_{n}$ for the $n$th Fibonacci number and $\widehat{\mathcal{F}}_{n}$ for the sequence $0,1, \widehat{\mathcal{F}}_{n}=2 \widehat{\mathcal{F}}_{n-1}+\widehat{\mathcal{F}}_{n-2}$. For $h$ and $\hat{h}$ in $\mathcal{H}_{n}$ associated to the sequence of digits $1,1, \ldots, 1$, and $2,2, \ldots, 2$, we get $a=\mathcal{F}_{n-2}, b=c=\mathcal{F}_{n-1}$, and $d=\mathcal{F}_{n}$, while $\hat{a}=\widehat{\mathcal{F}}_{n-2}, \hat{b}=\hat{c}=\widehat{\mathcal{F}}_{n-1}$, and $\hat{d}=\widehat{\mathcal{F}}_{n}$. We conclude by using $\lim _{n \rightarrow \infty} \mathcal{F}_{n} / \mathcal{F}_{n-1}=(1+\sqrt{5}) / 2$ and $\lim _{n \rightarrow \infty} \widehat{\mathcal{F}}_{n} / \widehat{\mathcal{F}}_{n-1}=(1+\sqrt{8}) / 2$.

From Theorem 1.1] one easily gets (see e.g. [2]):

Corollary 1.2. For every $0<\alpha<1$ there is $t_{0}$ so that for all $|t|>t_{0}$ and $\sigma$ close to 0 , we have $\left\|\left(I d-L_{s}\right)^{-1}\right\|_{1, t} \leq|t|^{\alpha}$.

Theorem 1.1 implies [5] section 4] exponential decay of correlations for $C^{1}$ observables and the absolutely continuous invariant probability (SRB) measure of the semi-flow $\phi^{t}$. We hope this will be a useful step towards proving exponential decay of correlations for (continuous-time) planar Sinai billiards, using [8]. (For the moment, only open continuous-time billiards have been considered [7, and they admit finite Markov sections.) See Remark 2.1 for extensions to other Gibbs states.

\section{Proof of Theorem 1.1}

We basically follow Dolgopyat's proof, as detailed in [5], 6], and [1. A key point is the Federer property of any absolutely continuous measure $\nu$ with continuous density bounded from above and from below: There exist $C, C^{\prime}>0$ so that if $I$, $J$ are adjacent intervals with $|I| \geq|J| / C$, then $\nu(I) \geq \nu(J) / C^{\prime}$. To exploit this information when considering $L_{\sigma}$ for $\sigma \neq 0$, the arguments in [3] (e.g. last lines of p. 367) and 11 (e.g. first lines of p. 43) use that there is $\alpha_{\sigma} \rightarrow 1$ when $\sigma \rightarrow 0$ so that $\widetilde{L}_{\sigma} f(x) \leq \alpha_{\sigma} \widetilde{L}_{0} f(x)$, for the normalised operators in (2.1) and positive $f$. The above inequality uses that there are finitely many branches and is for example not true for the Gauss map. To bypass this problem, we exploit carefully the Cauchy-Schwartz decomposition in Lemma 2.8 below (see also [2], Lemma 2).

Remark 2.1. Beware that even when there are finitely many branches, the Federer property is not true for arbitrary Gibbs measures $\nu$, in particular the measures $\nu_{\sigma}$ introduced below for $\sigma \neq 0$, contrary to what is stated in [3. Proposition 7]; [5] Lemma 6]; and [6, Lemma 4]. (Proposition 7 of [5] is true e.g. if $T$ is a $C^{2}$ circle map, and if $r$ is $C^{1}$ on the circle, and not only piecewise $C^{1}$. For a counterexample, take $T(x)=2 x$ modulo 1 with $\exp (r) \equiv 3$ on $[0,1 / 2]$ and $\exp (r) \equiv 3 / 2$ on $(1 / 2,1]$, and consider the intervals of size $1 / 2^{n}$ to the right and to the left of $1 / 2$. By adding $\epsilon \sin (2 \pi x)$ to $r$, this example can probably be made to satisfy the UNI condition [5] p. 537].) When there are finitely many branches, the Federer property does hold [4] 
for Gibbs measures and "most" adjacent intervals from the partitions in [3], [5], [6]: This is enough e.g. to recover the results in [3], in particular Theorem 1. When $\mathcal{H}$ is infinite, the situation is more complicated, but we expect that Theorem 1.1 will also hold for more general transfer operators $L_{s, g} f(x)=\sum_{T(y)=x} e^{-s r(y)} g(y) f(y)$ associated to suitable positive $g$.

Preliminary steps. Fix from now on $\hat{\rho}<\rho<1$. The inverse branches of $T^{n}$ satisfy $\left|h^{\prime \prime}\right| \leq \bar{K}\left|h^{\prime}\right|$ for all $n$ and the distortion constant $\bar{K}=K /(1-\rho)$, and similarly for $\left(r^{(n)}\right)^{\prime} \circ h$. As a consequence it is easy to prove that, for every $n \geq 1$, and each pair $h, k$ in $\mathcal{H}_{n}$, the function $\psi_{h, k}(x)=r^{(n)} \circ h-r^{(n)} \circ k$ satisfies $\sup \left|\psi_{h, k}^{\prime}\right| \leq 2 \bar{K}$. We next recall spectral properties of the $L_{s}$ (see e.g. [2] and references therein). Let $\sigma>\sigma_{0}$ be real. The essential spectral radius $\lambda_{\sigma}^{e}$ of $L_{\sigma}$ is strictly smaller than its spectral radius $\lambda_{\sigma}$ (in fact $\lambda_{\sigma}^{e} \leq \rho \lambda_{\sigma}$ ). Since $T$ is topologically mixing, the operator $L_{\sigma}$ has a unique (simple) eigenvalue $\lambda_{\sigma}$ of maximal modulus, for a strictly positive $C^{1}$ eigenfunction $f_{\sigma}$; the rest of the spectrum is in the disc of radius $\tau_{\sigma} \lambda_{\sigma}$ for some $\tau_{\sigma}<1$. The eigenvector $\mu_{\sigma}$ of $L_{\sigma}^{*}$ for $\lambda_{\sigma}$ is Lebesgue measure for $\sigma=0$, and for all $\sigma>\sigma_{0}$ is a positive Radon measure $\mu_{\sigma}$. We may assume $\mu_{\sigma}(1)=1$ and $\mu_{\sigma}\left(f_{\sigma}\right)=1$ so that $\nu_{\sigma}=f_{\sigma} \mu_{\sigma}$ is a probability measure. Note that $L_{\sigma}: C^{1}(I) \rightarrow C^{1}(I)$ depends continuously on $\sigma$, so that $\lambda_{\sigma}^{ \pm 1}, \tau_{\sigma}, f_{\sigma}^{ \pm 1}$, and $f_{\sigma}^{\prime}$ depend continuously on $\sigma$ (and therefore satisfy uniform bounds in any compact subset $\Sigma \subset\left(\sigma_{0}, \infty\right)$ ). Also, $\sigma \mapsto \lambda_{\sigma}$ is a nonincreasing function. Finally, the spectral radius of $L_{\sigma+i t}$ is not larger than $\lambda_{\sigma}$, and its essential spectral radius is not larger than $\rho \lambda_{\sigma}$ for all $t \in \mathbb{R}$.

It will be convenient to work with the normalised operators

$$
\widetilde{L}_{s}(f)=\frac{L_{s}\left(f_{\sigma} \cdot f\right)}{\lambda_{\sigma} f_{\sigma}}, \quad s=\sigma+i t .
$$

If $s=\sigma>\sigma_{0}$, the operator $\widetilde{L}_{\sigma}$ acting on $C^{1}(I)$ has spectral radius 1 , essential spectral radius $\leq \rho$, and fixes the constant function $\equiv 1$. Clearly $\widetilde{L}_{\sigma}^{*}$ preserves the probability measure $\nu_{\sigma}=f_{\sigma} \cdot \mu_{\sigma}$. Our starting point is a Lasota-Yorke inequality:

Lemma 2.2 (Lasota-Yorke). For every compact $\Sigma \subset\left(\sigma_{0}, \infty\right)$, there is a constant $C=C(\Sigma, \bar{K})>0$, so that for all $n \geq 1$, all $s \in \Sigma$ and all $f \in C^{1}(I)$ :

$$
\left|\left(\widetilde{L}_{s}^{n} f\right)^{\prime}(x)\right| \leq C(\Sigma, \bar{K})|s| \cdot \widetilde{L}_{\sigma}^{n}(|f|)(x)+\rho^{n} \cdot \widetilde{L}_{\sigma}^{n}\left(\left|f^{\prime}\right|\right)(x) .
$$

Lemma 2.2 indicates that we should concentrate on the sup component of the norm, which will be estimated by the $\mathcal{L}^{2}\left(d \nu_{0}\right)$ norm (see the beginning of the proof of Theorem 1.1 below). Indeed, the crucial estimate (Lemma 2.8) will show exponential decay of iterates of the operator in the $\mathcal{L}^{2}\left(d \nu_{0}\right)$ norm. These $\mathcal{L}^{2}\left(d \nu_{0}\right)$ integrals are oscillatory integrals in disguise: because of the weights $\exp (-s(r \circ h))$ in $L_{s}$, the integrand is the absolute value of a sum of complex numbers with strong phase variations for large $|t|$ (UNI is crucial here). We shall exhibit enough cancellations in the terms, via the key Lemma 2.4.

Proof. The Leibniz sum for the derivative of each term $\exp \left(-s\left(r^{(n)} \circ h\right)\right)\left|h^{\prime}\right| \cdot \frac{1}{\lambda_{\sigma} f_{\sigma}}$. $\left(f_{\sigma} f\right) \circ h$ forming $\left(\widetilde{L}_{s}^{n}(f)\right)^{\prime}\left(h \in \mathcal{H}_{n}\right)$ contains four terms. We can bound the first for all $s$ using our "distortion" assumption on $r$ since $|s|\left|\left(r^{(n)}\right)^{\prime} \circ h \| h^{\prime}\right| e^{-s(r \circ h)} \leq$ $|s| \bar{K} e^{-s(r \circ h)}$. The second one is controlled by the Renyi assumption on $T$. Compactness of $\Sigma$ and continuity of $\sigma \mapsto \lambda_{\sigma}$ and $\sigma \mapsto f_{\sigma} \operatorname{imply}_{\sup _{\sigma \in \Sigma}}\left|f_{\sigma}^{\prime}\right|<\infty$ and $\inf _{\sigma \in \Sigma} f_{\sigma}>0$, so that the third term may be controlled by $\frac{\left|f_{\sigma}^{\prime}\right|}{\lambda_{\sigma} f_{\sigma}^{2}} \leq C_{\Sigma} \frac{1}{\lambda_{\sigma} f_{\sigma}}$ for 
some $C_{\Sigma}>0$. Finally, the last term can be estimated using

$$
\left|\left(f_{\sigma} \cdot f\right)^{\prime} \circ h\right|\left|h^{\prime}\right| \leq \rho^{n}\left[\left|f_{\sigma}^{\prime} \cdot f\right| \circ h+\left(f_{\sigma} \cdot\left|f^{\prime}\right|\right) \circ h\right] .
$$

We can ensure $\bar{K}|s|+2 C_{\Sigma}+2 \rho C_{\Sigma} \leq C(\Sigma, \bar{K})|s|$ (for fixed $\Sigma$, if $|s|$ is large, i.e., if $|t|$ is large enough, then $C(\Sigma, \bar{K})$ is close to $\bar{K})$.

We next state and prove an elementary lemma about complex numbers with almost opposite phases. Note that $2 / 3<(\sqrt{7}-1) / 2<1$.

Lemma 2.3 (Calculus lemma). For each $\eta \in[(\sqrt{7}-1) / 2,1)$ and every pair of complex numbers, $z_{1}=r_{1} \exp \left(i \theta_{1}\right)$ and $z_{2}=r_{2} \exp \left(i \theta_{2}\right)$,

$$
\cos \left(\theta_{1}-\theta_{2}\right) \leq 1 / 2 \Rightarrow\left|z_{1}+z_{2}\right| \leq \max \left(\eta r_{1}+r_{2}, r_{1}+\eta r_{2}\right) .
$$

Proof. Up to exchanging $z_{1}$ and $z_{2}$, we can suppose that $r_{1} \leq r_{2}$ so that $\eta r_{1}+r_{2} \geq$ $r_{1}+\eta r_{2}$. Our assumption on $\cos \left(\theta_{1}-\theta_{2}\right)$ implies

$$
\left|z_{1}+z_{2}\right|^{2}=r_{1}^{2}+r_{2}^{2}+2 r_{1} r_{2} \cos \left(\theta_{1}-\theta_{2}\right) \leq r_{1}^{2}+r_{2}^{2}+r_{1} r_{2} .
$$

Since $\left(\eta r_{1}+r_{2}\right)^{2}=\eta^{2} r_{1}^{2}+r_{2}^{2}+2 \eta r_{1} r_{2}$, we must show $r_{1}^{2}\left(1-\eta^{2}\right)+2 r_{1} r_{2}(1 / 2-\eta) \leq 0$.

Since $\eta-1 / 2 \geq 1-\eta^{2} \geq 0$ (use $3 \geq \sqrt{7} \geq 2$ ), we get

$r_{1}^{2}\left(1-\eta^{2}\right)+2 r_{1} r_{2}(1 / 2-\eta) \leq r_{1}^{2}(\eta-1 / 2)+r_{1} r_{2}(1 / 2-\eta) \leq r_{1}(\eta-1 / 2)\left(r_{1}-r_{2}\right) \leq 0$.

Preparatory lemmas in view of $\mathcal{L}^{2}$ contraction. In the next lemma, we combine $U N I$ and Lemma 2.3 to obtain cancellation-type estimates on terms appearing when applying iterates of $\widetilde{L}_{\sigma}$ to a suitable pair $(u, v)$ of initial test functions in $C^{1}(I)$. We first introduce the "cone" condition that $(u, v)$ must satisfy: there exist $C>0$ and $t \in \mathbb{R}$ so that

$$
u>0, \quad 0 \leq|v| \leq u, \quad \max \left(\left|u^{\prime}(x)\right|,\left|v^{\prime}(x)\right|\right) \leq 2 C|t| u(x) .
$$

Lemma 2.4 (Exhibiting cancellations). Assume that UNI holds for $D$ and $n_{0}$. Then, for all $C>0$, there exist $n_{1} \geq n_{0}, \delta>0$ and $\Delta>0$, so that for any $|t|>2 \pi / D$, and all $u, v \in C^{1}(I)$ satisfying (2.4) for $C$ and $t$, we have the following:

Fix $n \geq n_{1}$, and let $h, k \in \mathcal{H}_{n}$ be the branches from UNI. For every $x_{0} \in I$, there is $x_{1} \in I$ with $\left|x_{0}-x_{1}\right|<\Delta /|t|$, so that the function

$\left.F(x):=e^{-(\sigma+i t) r^{(n)}(h(x))}\left|h^{\prime}(x)\right|\left(\left(v \cdot f_{\sigma}\right) \circ h\right)(x)+e^{-(\sigma+i t) r^{(n)}(k(x))} \mid k^{\prime}(x)\right]\left(\left(v \cdot f_{\sigma}\right) \circ k\right)(x)$ satisfies for all $x$ s.t. $\left|x-x_{1}\right|<\delta /|t|$, all $\sigma>\sigma_{0}$, and all $\eta>(\sqrt{7}-1) / 2$,

$$
\begin{aligned}
& |F(x)| \leq \max \left[\eta e^{-\sigma r^{(n)}(h(x))}\left|h^{\prime}(x)\right|\left(u \cdot f_{\sigma}\right) \circ h\right)(x) \\
& \quad+e^{-\sigma r^{(n)}(k(x))}\left|k^{\prime}(x)\right|\left(\left(u \cdot f_{\sigma}\right) \circ k\right)(x), \\
& \left.\left.\left.\quad e^{-\sigma r^{(n)}(h(x))}\left|h^{\prime}(x)\right|\left(u \cdot f_{\sigma}\right) \circ h\right)(x)+\eta e^{-\sigma r^{(n)}(k(x))}\left|k^{\prime}(x)\right|\left(\left(u \cdot f_{\sigma}\right) \circ k\right)(x)\right)\right] .
\end{aligned}
$$

When the maximum in (2.5) is attained by the expresssion where the $\eta$ factor is attached to branch $h$ we say we are "in case $h$," and otherwise "in case k."

It follows from the proof that $n_{1} \geq n_{0}$ so that $3 \times 16 C \rho^{n_{1}}<1 / 24$ works. In the application of Lemma 2.4 in Lemma [2.7] we require $C \geq C(\Sigma, \bar{K})$ from Lemma 2.2 
Proof. Let us fix $x_{0} \in I$. Assume first (this case does not require UNI) that

$$
\left|v\left(h\left(x_{0}\right)\right)\right| \leq \max \left(u\left(h\left(x_{0}\right)\right) / 2, u\left(k\left(x_{0}\right)\right) / 2\right) .
$$

Let us suppose the maximum is realised for $u \circ h$ (the other case is symmetric). Then it is easy to see that for any $\epsilon>0$, if $\left|x-x_{0}\right|<\delta_{1} /|t|$, with $\delta_{1}\left(2 C \rho^{n_{0}}\right)=\epsilon$, we have $\exp (-\epsilon) \leq \frac{u(h(x))}{u\left(h\left(x_{0}\right)\right)} \leq \exp (\epsilon) .\left(\right.$ Use $\exp \left(\log u(h(x))-\log u\left(h\left(x_{0}\right)\right)\right) d x \leq$ $\exp \int_{h(x)}^{h\left(x_{0}\right)}\left|(\log u(y))^{\prime}\right| d y$, the assumed bound on $\left|u^{\prime}\right| / u$ from (2.4) and $n \geq n_{0}$.)

To prove (2.5), it is then enough to check that $\left|x-x_{0}\right|<\delta_{1} /|t|$ implies $|v(h(x))| \leq$ $\eta^{\prime} u\left(h\left(x_{0}\right)\right)$ for some $\eta^{\prime}>2 / 3$ with $\eta^{\prime} \exp (\epsilon) \leq \eta$ : indeed, we would then have $|v(h(x))| \leq \eta^{\prime} \exp (\epsilon) u(h(x)) \leq \eta u(h(x))$ whenever $\left|x-x_{0}\right|<\delta_{1} /|t|$, so that (2.5) would hold. Assume for a contradiction that no such $\eta^{\prime}$ exists, i.e., for each $2 / 3<$ $\eta^{\prime} \leq \eta \exp (-\epsilon)$ there is $x_{1}$ with $\left|x_{1}-x_{0}\right| \leq \delta_{1} /|t|$ and $\left|v\left(h\left(x_{1}\right)\right)\right| \geq \eta^{\prime}\left(u\left(h\left(x_{0}\right)\right)\right)$, so that (use (2.6) $)\left|v\left(h\left(x_{0}\right)\right)-v\left(h\left(x_{1}\right)\right)\right| \geq\left(\eta^{\prime}-1 / 2\right) u\left(h\left(x_{0}\right)\right)$. On the other hand, (2.4) and the choice of $\epsilon$ imply that there is $y$ with $\left|y-x_{0}\right| \leq \delta_{1} /|t|$ so that

$$
\left|v\left(h\left(x_{0}\right)\right)-v\left(h\left(x_{1}\right)\right)\right| \leq u(h(y)) 2 C|t| \rho^{n_{0}} \frac{\delta_{1}}{|t|} \leq u\left(h\left(x_{0}\right)\right) e^{\epsilon} 2 C \rho^{n_{0}} \delta_{1}=u\left(h\left(x_{0}\right)\right) \epsilon e^{\epsilon},
$$

a contradiction if $\epsilon \exp (\epsilon)<1 / 6$. This ends the easy case, where we can take $x_{1}=x_{0}$ (i.e. $\left.\Delta_{1}=0\right)$ and $\delta_{1}=\epsilon /\left(2 C \rho^{n_{0}}\right)$ for small (independently of $u, v, C$, etc.) $\epsilon>0$. (The dependence of $\delta_{1}$ on $C$ can be removed by taking large enough $n$.)

Let us now move to the more interesting situation when

$$
\left|v\left(h\left(x_{0}\right)\right)\right|>\max \left(u\left(h\left(x_{0}\right)\right) / 2, u\left(k\left(x_{0}\right)\right) / 2\right) .
$$

We shall use $U N I$ to show that we are in a position to apply Lemma 2.3 to the sum forming $F(x)$, for $x$ in a $\delta_{2} /|t|$-interval around a point $x_{1}$ that is $\Delta_{2} /|t|$ close to $x_{0}$. Since $f_{\sigma}$ is real and positive, the difference $\theta(x)$ between the argument of the two terms of $F(x)$ can be decomposed as $\theta(x)=t \psi_{h, k}(x)+\arg (v(h(x)))-\arg (v(k(x)))$.

Let us first show the claim by assuming that we found $\delta_{2}, \Delta_{2}$ so that $\cos \theta(x) \leq$ $1 / 2$, for all $x$ with $\left|x-x_{1}\right| \leq \delta_{2} /|t|$, for some $x_{1}$ with $\left|x_{1}-x_{0}\right|<\Delta_{2} /|t|$, leaving the (nontrivial) proof of this fact for the end. We have

$$
r_{1}(x)=e^{-\sigma r^{(n)}(h(x))}\left|h^{\prime}(x)\right|\left(|v| \cdot f_{\sigma}\right)(h(x))
$$

and

$$
r_{2}(x)=e^{-\sigma r^{(n)}(k(x))}\left|k^{\prime}(x)\right|\left(|v| \cdot f_{\sigma}\right)(k(x)) .
$$

Fix $x$ with $\left|x-x_{1}\right| \leq \delta_{2} /|t|$, and assume (the other case is analogous) that $r_{1}(x) \leq$ $r_{2}(x)$. Lemma 2.3 then yields the claim:

$$
\begin{aligned}
|F(x)| & \leq \eta e^{-\sigma r^{(n)}(h(x))}\left|h^{\prime}(x)\right|\left(|v| \cdot f_{\sigma}\right)(h(x))+e^{-\sigma r^{(n)}(k(x))}\left|k^{\prime}(x)\right|\left(|v| \cdot f_{\sigma}\right)(k(x)) \\
& \leq \eta e^{-\sigma r^{(n)}(h(x))}\left|h^{\prime}(x)\right|\left(u \cdot f_{\sigma}\right)(h(x))+e^{-\sigma r^{(n)}(k(x))}\left|k^{\prime}(x)\right|\left(u \cdot f_{\sigma}\right)(k(x)) .
\end{aligned}
$$

It remains to prove that $\cos \theta(x) \leq 1 / 2$ for $x$ as above and some $\delta_{2}, \Delta_{2}$. For this, the following consequence of (2.7) and (2.4) will be helpful: for all $y, z$ with $\left|z-x_{0}\right| \leq\left|y-x_{0}\right| \leq \xi / \mid t$,

$$
\begin{aligned}
|v(h(y))| & \geq\left|v\left(h\left(x_{0}\right)\right)\right|-\left|v\left(h\left(x_{0}\right)\right)-v(h(y))\right| \\
& \geq u\left(h\left(x_{0}\right)\right) / 2-\rho^{n_{0}} \frac{\xi}{|t|} 2 C|t| u(h(z)) \\
& \geq u\left(h\left(x_{0}\right)\right)\left(1 / 2-\exp (\epsilon) \rho^{n_{0}} \xi 2 C\right) \geq u\left(h\left(x_{0}\right)\right) / 4 .
\end{aligned}
$$


Next observe that, because of (2.4), (2.7), $V(x)=\arg (v(h(x))-\arg (v(k(x))$ does not vary too much around $x_{0}$. More precisely:

$$
\begin{aligned}
\left|V(x)-V\left(x_{0}\right)\right| & =\mid \log [v(h(x)) / v(k(x))]-\log \left[v\left(h\left(x_{0}\right)\right) / v\left(k\left(x_{0}\right)\right)\right] \\
& \leq\left|\log \left[\frac{v(h(x))}{v\left(h\left(x_{0}\right)\right)}\right]\right|+\left|\log \left[\frac{v\left(k\left(x_{0}\right)\right)}{v(k(x))}\right]\right|,
\end{aligned}
$$

and, if $\left|x-x_{0}\right| \leq \xi /|t|$,

$$
\left|\log \left[\frac{v(h(x))}{v\left(h\left(x_{0}\right)\right)}\right]\right| \leq\left|h(x)-h\left(x_{0}\right)\right| \frac{\left|v^{\prime}(h(y))\right|}{|v(h(y))|} \leq \rho^{n} \frac{\xi}{|t|} 8 C|t| e^{\epsilon} \frac{u\left(h\left(x_{0}\right)\right)}{u\left(h\left(x_{0}\right)\right)} \leq \xi 8 C e^{\epsilon} \rho^{n} .
$$

(We used $\left|y-x_{0}\right| \leq\left|x-x_{0}\right|$ and (2.8), (2.7).) We may control $\left|\log \left[\frac{v\left(k\left(x_{0}\right)\right)}{v(k(x))}\right]\right|$, mutatis mutandis, and we have for $\left|x-x_{0}\right|<\xi /|t|$ :

$$
\left|V(x)-V\left(x_{0}\right)\right| \leq \xi 16 C \exp (\epsilon) \rho^{n} .
$$

Recall that we have to show $\cos \theta(x) \leq 1 / 2$ in a suitable interval. We first find $x_{1}$ with $\left|x_{1}-x_{0}\right|<\Delta_{2} /|t|$ such that $\left|\theta\left(x_{1}\right)-\pi\right| \leq \pi / 24$. For this, we use $U N I$ which ensures that, since $t\left(\psi(z)-\psi\left(x_{0}\right)\right)=t\left(z-x_{0}\right) \psi^{\prime}(y)$ for $y \in I$, if $\Delta_{2}=2 \pi / D$, then $\left\{t\left(\psi(z)-\psi\left(x_{0}\right)\right) \bmod 2 \pi|| z-x_{0}\left|\leq \Delta_{2} /\right| t \mid\right\}=[0,2 \pi)$. (We use here $|t|>2 \pi / D$.) In particular, there is $z=x_{1}$ so that $t\left(\psi\left(x_{1}\right)-\psi\left(x_{0}\right)\right)=\pi-\theta\left(x_{0}\right)(\bmod 2 \pi)$. Applying (2.10) to $x=x_{1}, \xi=\Delta_{2}$, we find

$$
\begin{aligned}
\left|\theta\left(x_{1}\right)-\pi\right| & =\left|\theta\left(x_{0}\right)+t\left(\psi\left(x_{1}\right)-\psi\left(x_{0}\right)\right)+\left(V\left(x_{1}\right)-V\left(x_{0}\right)\right)-\pi\right| \\
& \leq\left|V\left(x_{1}\right)-V\left(x_{0}\right)\right|<\Delta_{2} 16 C \exp (\epsilon) \rho^{n}<\pi / 24,
\end{aligned}
$$

if $n$ is large enough (depending on $C$ and, via $\Delta_{2}$, on $D$ ).

To conclude, we apply (2.10) and the "distortion" upper bound, using $\left|x-x_{0}\right|<$ $\left|x-x_{1}\right|+\left|x_{1}-x_{0}\right|<\left(\delta_{2}+\Delta_{2}\right) /|t|$ and $\left|x-x_{1}\right|<\Delta_{2} /|t|$ to get, if $n$ is large enough (depending on $C$ and $D$ ) and $0<\delta_{2} \leq \Delta_{2}$ is small enough (depending on $\bar{K}$ ):

$$
\begin{aligned}
|\theta(x)-\pi| & \leq \pi / 24+\left|\theta(x)-\theta\left(x_{1}\right)\right| \\
& \leq \pi / 24+|t|\left|\psi(x)-\psi\left(x_{1}\right)\right|+\left|V(x)-V\left(x_{0}\right)\right|+\left|V\left(x_{1}\right)-V\left(x_{0}\right)\right| \\
& \leq \pi / 24+2 \bar{K}|t| \frac{\delta_{2}}{|t|}+16 C \exp (\epsilon) \rho^{n} D|t| \frac{\delta_{2}+2 \Delta_{2}}{|t|}<\pi / 12 .
\end{aligned}
$$

Taking $\delta=\min \left(\delta_{1}, \delta_{2}\right)$ and $\Delta=\Delta_{2}$, we have proved the lemma.

Remark 2.5. If we replace $U N I$ by the assumption that there exist $D>0, n_{0}$, and two inverse branches $h$ and $k$ of $T^{n_{0}}$ so that inf $\left|\psi_{h, k}^{\prime}\right| \geq D$, then for every $n \geq n_{0}$ there are $\hat{h}, \hat{k} \in \mathcal{H}_{n}$ so that inf $\left|\psi_{\hat{h}, \hat{k}}^{\prime}\right| \geq \rho^{n-n_{0}} D$. (Take $\hat{h}=h \circ \ell, \hat{k}=k \circ \ell$, for $\ell \in \mathcal{H}_{n-n_{0}}$ and observe that $\psi_{\hat{h}, \hat{k}}(x)=\psi_{h, k}(\ell(x))$.) However, this is not enough. In (2.11) we would get (in view of the definition of $\Delta_{2}$ ) $\frac{2 \pi}{D \rho^{n-n_{0}}} 8 C \exp (\epsilon) \rho^{n}=$ $\frac{16 C}{D} \exp (\epsilon) \rho^{n_{0}}$, which is independent of $n$ and not necessarily smaller than $\pi / 24$. (The constant 16 can be reduced, but not below 1.) Unfortunately, the strategy presented on p. 545 of [5] seems to suffer from the same problem.

The following consequence of Lemma 2.4 will be instrumental in arriving at Lemma 2.8

Corollary 2.6. Let $T$ satisfy UNI for D. Let $C>0$, and let $n_{1}=n_{1}(C), \delta=\delta(C)$, $\Delta=\Delta(C)$ be given by Lemma 2.4. Fix $n \geq n_{1}$, let $h, k \in \mathcal{H}_{n}$ come from UNI, and let $\rho_{n, C}=\min \left(\min \left|h^{\prime}\right|, \min \left|k^{\prime}\right|\right)$ (we have $\left.0<\rho_{n, C} \leq \rho^{n}\right)$. 
Then for every $|t|>2 \pi / D$, every $u, v \in C^{1}(I)$ satisfying (2.4) for $C$ and $|t|$, and each $\eta>(\sqrt{7}-1) / 2$ (recall Lemma 2.3), there are:

- a finite set of (disjoint) intervals $\left[a_{j}, b_{j+1}\right]=I_{j} \subset I, j=0, \ldots, N-1$, with $\left|I_{j}\right| \geq \delta /|t|, a_{0} \leq \Delta /|t|$, and $b_{N} \geq 1-\Delta /|t|$; also, setting $J_{j}=\left[b_{j}, a_{j}\right]$, we have $0<\left|J_{j}\right| \leq 2 \Delta /|t|$; to each $I_{j}$ is associated type $\left(I_{j}\right) \in\{h, k\}$; we write $\hat{I}_{j}$ for the middle third interval of $I_{j}$;

- a function $\chi=\chi(u, v, n, \eta) \in C^{1}(I)$ (in particular, $\chi$ depends on $C,|t|$ ) so that:

$$
\left\{\begin{array}{l}
\eta \leq \chi(x) \leq 1, \quad\left|\chi^{\prime}\right| \leq \frac{3(1-\eta)}{\rho_{n, C} \delta}|t|, \\
\operatorname{type}\left(I_{j}\right)=h \text { and } x \in \hat{I}_{j} \Rightarrow \chi_{h(x)}=\eta, \\
\operatorname{type}\left(I_{j}\right)=k \text { and } x \in \hat{I}_{j} \Rightarrow \chi_{k(x)}=\eta, \\
\chi(y)<1 \Rightarrow\left[y=h(x), x \in I_{j}, \operatorname{type}\left(I_{j}\right)=h\right] \text { or }\left[y=k(x), x \in I_{j}, \operatorname{type}\left(I_{j}\right)=k\right] .
\end{array}\right.
$$

Finally, for $s=\sigma+i t$ with $\sigma>\sigma_{0}$, we have $\left|\widetilde{L}_{s}^{n}(v)(x)\right| \leq \widetilde{L}_{\sigma}^{n}(\chi u)(x), \forall x \in I$.

Note that $\sup \left|\chi^{\prime}\right| /|t|$ can be made arbitrarily small by taking $\eta<1$ close to 1 , once $C$ and $h, k, n$ are fixed. To exploit Corollary 2.6 we shall use the following:

Lemma 2.7 (Invariance of "cone condition"). Let T satisfy UNI for $D$ and fix $\Sigma$ a compact subset of $\left(\sigma_{0}, \infty\right)$. Let $C(\Sigma, \bar{K})$ be from Lemma 2.2 and fix $C>1$ so that: $C \geq C(\Sigma, \bar{K}) \cdot \max \left(1, \max _{\sigma \in \Sigma}|\sigma| D /(2 \pi)\right)$.

Then, there is $n_{2} \geq n_{1}$ ( $n_{1}$ from Lemma 2.4) so that for every large enough $|t|>$ $2 \pi / D$, each $u$, $v$, satisfying (2.4) for $C$ and $t$, and all $n \geq n_{2}$, taking $\eta=\eta(n)<1$ close enough to 1 , and $\chi=\chi(u, v, \eta)$ from Corollary 2.6 the pair $\hat{u}=\widetilde{L}_{\sigma}^{n}(\chi u)$, $\hat{v}=\widetilde{L}_{s}^{n}(v)$, satisfies (2.4), for the same $|t|$ and $C$, and for all $s=\sigma+i$ with $\sigma \in \Sigma$.

Proof. Corollary 2.6 says that $|\hat{v}(x)|=\left|\widetilde{L}_{s}^{n}(v)(x)\right| \leq \widetilde{L}_{\sigma}^{n}(\chi u)(x)=\hat{u}(x)$ for all $x \in I$. We also have $\inf \hat{u}>0$ since $\inf (\chi u)>0$ and $\widetilde{L}_{\sigma}$ preserves the cone of strictly positive functions. To check the condition on $\max \left(\left|\hat{u}^{\prime}\right|,\left|\hat{v}^{\prime}\right|\right)$ we shall (finally!) invoke the Lasota-Yorke inequality from Lemma 2.2 (recalling also that $\widetilde{L}_{\sigma}$ is normalised so that $\left.\sup \widetilde{L}_{\sigma}|f| \leq \sup |f|\right)$. We first consider $\hat{u}^{\prime}$ and get, using $\left|u^{\prime}\right| \leq 2 C|t| u, \chi \geq \eta$ and $\left|\chi^{\prime}\right| \leq 1(\eta=\eta(C, n)$ is close to 1$)$ :

$$
\begin{aligned}
\left|\frac{d}{d x} \widetilde{L}_{\sigma}^{n}(\chi u)(x)\right| & \leq C(\Sigma, \bar{K}) \sigma \widetilde{L}_{\sigma}^{n}(\chi u)(x)+\rho^{n} \widetilde{L}_{\sigma}^{n}\left(\left|\chi^{\prime} u+\chi u^{\prime}\right|\right)(x) \\
& \leq\left(C(\Sigma, \bar{K})|t|+\rho^{n}\left(\frac{1}{\eta}+2 C|t|\right)\right) \widetilde{L}_{\sigma}^{n}(\chi u)(x) \leq 2 C|t| \widetilde{L}_{\sigma}^{n}(\chi u)(x),
\end{aligned}
$$

if $n \geq n_{2} \geq n_{1}$ and $C \geq C(\Sigma, \bar{K})$.

The computation for $\left|\hat{v}^{\prime}\right|$ is similar:

$$
\begin{aligned}
\left|\frac{d}{d x} \widetilde{L}_{s}^{n}(v)(x)\right| & \leq C(\Sigma, \bar{K})|s| \widetilde{L}_{\sigma}^{n}(|v|)(x)+\rho^{n} \widetilde{L}_{\sigma}^{n}\left(\left|v^{\prime}\right|\right)(x) \\
& \leq \frac{C(\Sigma, \bar{K})|s|+2 C|t| \rho^{n}}{\eta} \widetilde{L}_{\sigma}^{n}(\chi u)(x) \leq 2 C|t| \widetilde{L}_{\sigma}^{n}(\chi u)(x),
\end{aligned}
$$

if $n \geq n_{2} \geq n_{1}$ and $C(\Sigma, \bar{K})|s| \leq C|t|$.

Proof of the $\mathcal{L}^{2}$ contraction and proof of Theorem 1.1, We shall see below that the case $\sup \left|f^{\prime}\right|>2 C|t| \sup |f|$ is easy. We next prove the key " $\mathcal{L}^{2}$ contraction lemma" (see [3] Lemma 4]) to handle the other case. 
Lemma $2.8\left(\mathcal{L}^{2}\left(\nu_{1}\right)\right.$ contraction). Assume UNI. Let $\Sigma, C, n \geq n_{2},|t|>2 \pi / D$, be as in Lemma 2.7. There is $\beta<1$ so that for all $\sigma$ close enough to 0 , and for all $0 \neq f \in C^{1}$ with $\sup \left|f^{\prime}\right| \leq 2 C|t| \sup |f|$,

$$
\int\left|\widetilde{L}_{\sigma+i t}^{m n} f\right|^{2} d \nu_{0}<\beta^{m} \sup |f|^{2}, \forall m \geq 1
$$

Proof. Recall $\eta<1$ was taken close to 1 in Lemma 2.7. For $s=\sigma+i t$ with $\sigma \in \Sigma$, define a sequence of pairs $\left(u_{m}, v_{m}\right), m \geq 0$, of functions in $C^{1}(I)$ :

$$
u_{0} \equiv 1, v_{0}=\frac{f}{\sup |f|}, \chi_{0}=\chi_{u_{0}, v_{0}, n}, \quad u_{m}=\widetilde{L}_{\sigma}^{n}\left(\chi_{m-1} u_{m-1}\right), \quad v_{m}=\widetilde{L}_{s}^{n}\left(v_{m-1}\right) .
$$

Lemma2.7 implies that all $\left(u_{m}, v_{m}\right)$ satisfy (2.4) for $C, t$, and all $m$. (Note also that $u_{m} \leq 1$ for all $m$.) In particular, $\left|\widetilde{L}_{s}^{m n}(f / \sup |f|)\right|=\left|v_{m}\right| \leq u_{m}$, and to prove the lemma, it is enough to show that there is $\beta_{1}<1$, so that $\int u_{m+1}^{2} d \nu_{0} \leq \beta_{1} \int u_{m}^{2} d \nu_{0}$ for all $m$ (note that $\int u_{0}^{2} d \nu_{0}=1$ ).

The definition of $u_{m+1}$ and the Cauchy-Schwartz inequality imply

$$
\begin{aligned}
\lambda_{\sigma}^{2 n} f_{\sigma}^{2}(x) u_{m+1}^{2}(x)= & \left(\sum_{\ell \in \mathcal{H}_{n}} e^{-\sigma r^{(n)}(\ell(x))}\left|\ell^{\prime}(x)\right|\left(\chi_{m} \cdot f_{\sigma} \cdot u_{m}\right)(\ell(x))\right)^{2} \\
\leq & \max _{I} \frac{f_{\sigma}}{f_{0}} \sum_{\ell \in \mathcal{H}_{n}}\left|\ell^{\prime}(x)\right|\left(f_{0} \cdot u_{m}^{2}\right)(\ell(x)) \\
& \quad \max _{I} \frac{f_{\sigma}}{f_{2 \sigma}} \sum_{\ell \in \mathcal{H}_{n}} e^{-2 \sigma r^{(n)}(\ell(x))}\left|\ell^{\prime}(x)\right|\left(\chi_{m}^{2} \cdot f_{2 \sigma}\right)(\ell(x)) .
\end{aligned}
$$

Now, if $x \in \hat{I}_{j}$ for $\chi_{m}$, of type $h$, say (type $k$ is similar), we get

$$
\begin{aligned}
\frac{1}{\lambda_{2 \sigma}^{n} f_{2 \sigma}(x)} & \sum_{\ell \in \mathcal{H}_{n}} e^{-2 \sigma r^{(n)} \ell(x)}\left|\ell^{\prime}(x)\right|\left(\chi_{m}^{2} \cdot f_{2 \sigma}\right)(\ell(x)) \\
& \leq 1-\left(1-\eta^{2}\right) e^{-2 \sigma r^{(n)}(h(x))}\left|h^{\prime}(x)\right| \frac{f_{2 \sigma}(h(x))}{\lambda_{2 \sigma}^{n} f_{2 \sigma}(x)} \leq 1-\epsilon\left(1-\eta^{2}\right)=\eta^{\prime}<1
\end{aligned}
$$

(we used $e^{-2 \sigma r^{(n)}(h(x))}\left|h^{\prime}(x)\right| f_{2 \sigma}(h(x)) /\left(\lambda_{2 \sigma}^{n} f_{2 \sigma}(x)\right) \geq \epsilon>0$ if $n$ and $h$ are fixed; obviously, $\eta^{\prime}$ depends on $n$ ). Denote

$$
\xi(\sigma, n)=\frac{\lambda_{2 \sigma}^{n} f_{0}(x) f_{2 \sigma}(x)}{\lambda_{\sigma}^{2 n} f_{\sigma}^{2}(x)} \cdot \max _{I} \frac{f_{\sigma}}{f_{2 \sigma}} \cdot \max _{I} \frac{f_{\sigma}}{f_{0}} .
$$

We showed that for some $\eta^{\prime}<1$ and all $x \in \bigcup_{j} \hat{I}_{j}$ (recall $\lambda_{0}=1$ ),

$$
u_{m+1}^{2}(x) \leq \eta^{\prime} \xi(\sigma, n) \widetilde{L}_{0}^{n}\left(u_{m}^{2}\right)(x) .
$$

If $x \notin \bigcup_{j} \hat{I}_{j}$, the Cauchy-Schwartz inequality just gives, since $\chi_{m} \leq 1$,

$$
u_{m+1}^{2}(x) \leq \xi(\sigma, n) \widetilde{L}_{0}^{n}\left(u_{m}^{2}\right)(x) .
$$

We claim that there is $\hat{\delta}$, independent of $m, n$, and $t$, so that if $\hat{J}_{j}$ is the union of the rightmost third of $I_{j}, J_{j}$, and the leftmost third of $\hat{I}_{j+1}$, then

$$
\int_{\hat{I}_{j}} \widetilde{L}_{0}^{n}\left(u_{m}^{2}\right) d \nu_{0} \geq \hat{\delta} \cdot \int_{\hat{J}_{j}} \widetilde{L}_{0}^{n}\left(u_{m}^{2}\right) d \nu_{0}
$$


We finish the proof assuming (2.15): if $\hat{\delta}\left(\beta_{2}-\eta^{\prime}\right) \geq\left(1-\beta_{2}\right)\left(\right.$ e.g. $\left.\beta_{2}=: \frac{1+\eta^{\prime} \hat{\delta}}{1+\hat{\delta}}<1\right)$,

$$
\begin{aligned}
\int_{I} u_{m+1}^{2} d \nu_{0} & \leq \xi(\sigma, n) \sum_{j}\left(\eta^{\prime} \int_{\hat{I}_{j}} \widetilde{L}_{0}^{n}\left(u_{m}^{2}\right) d \nu_{0}+\int_{\hat{J}_{j}} \widetilde{L}_{0}^{n}\left(u_{m}^{2}\right) d \nu_{0}\right) \\
& \leq \xi(\sigma, n) \beta_{2}\left(\sum_{j} \int_{\hat{I}_{j}} \widetilde{L}_{0}^{n}\left(u_{m}^{2}\right) d \nu_{0}+\int_{\hat{J}_{j}} \widetilde{L}_{0}^{n}\left(u_{m}^{2}\right) d \nu_{0}\right) \\
& =\xi(\sigma, n) \beta_{2} \int_{I} \widetilde{L}_{0}^{n}\left(u_{m}^{2}\right) d \nu_{0}=\xi(\sigma, n) \beta_{2} \int_{I} u_{m}^{2} d \nu_{0} .
\end{aligned}
$$

(In the last line we used that the dual of $\widetilde{L}_{0}^{n}$ leaves $\nu_{0}$ fixed.) By taking $\sigma$ sufficiently close to 0 (depending on $n$, which is fixed) we can assume that $\xi(\sigma, n) \cdot \beta_{2}<1$.

It remains to show (2.15). It suffices to prove that $\int_{\hat{I}_{j}} w^{2} d \nu_{0} \geq \hat{\delta} \int_{\hat{J}_{j}} w^{2} d \nu_{0}$ for all $C^{1}$ functions $w$ with $\sup w \leq 1$ and $\left|w^{\prime}(z)\right| \leq 2 C|t| w(z)$ (recall Lemma 2.7 and use Lemma 2.2 and $\left.\widetilde{L}_{0} 1 \equiv 1\right)$. Note that such $w$ satisfy, for all $x \in \hat{I}_{j}, y \in \hat{J}_{j}$ :

$$
\frac{w^{2}(y)}{w^{2}(x)}=\exp 2(\log w(x)-\log w(y))=\exp 2 \int_{x}^{y}\left(w^{\prime} / w\right)(z) d z \leq \exp (4 C(2 \Delta+\delta)) .
$$

Applying the above inequality, and making use of the Federer property (for intervals with length-ratio $3 \Delta$ ), of $\nu_{0}$ which has density $f_{0}$ (bounded from above and from below) with respect to Lebesgue measure, we find

$$
\int_{\hat{I}_{j}} w^{2} d \nu_{0} \geq \nu_{0}\left(\hat{I}_{j}\right) \min _{\hat{I}_{j}} w^{2} \geq \tilde{\delta} e^{-(4 C(2 \Delta+\delta))} \nu_{0}\left(\hat{J}_{j}\right) \max _{\hat{J}_{j}} w^{2} \geq \hat{\delta} \int_{\hat{J}_{j}} w^{2} d \nu_{0} .
$$

We are finally ready to prove the theorem:

Proof. Since there exists $B$ so that ( $\lambda_{\sigma}$ is simple) $\left\|L_{s}^{n}\right\|_{1, t} \leq B \lambda_{\sigma}^{n}\left\|\widetilde{L}_{s}^{n}\right\|_{1, t}$ for all $n \geq 1$, and since $\lambda_{0}=1$ and $\sigma$ is in a neighbourhood of 0 , it is enough to show that there exist $\widetilde{A}$ and $\tilde{\gamma}<1$ so that $\left\|\widetilde{L}_{s}^{n}\right\|_{1, t} \leq \tilde{\gamma}^{n}$, for $n \geq \widetilde{A} \log |t|$. Clearly, this will follow from the existence of $n_{4}$ and $\widehat{A}$ so that $\left\|\widetilde{L}_{s}^{n_{4} m}\right\|_{1, t} \leq \tilde{\gamma}^{n_{4} m}$ for all $m \geq \widehat{A} \log |t|$ (write $n=q n_{4}+r$, with $q, r \in \mathbb{Z}^{+}$and $0 \leq r<n_{4}$, and use $\left\|\widetilde{L}_{s}^{q}\right\|_{1, t} \leq \widetilde{B}$ ).

Let (see Lemma 2.7) $C=\max (3 / 2, C(\Sigma, \bar{K}) \cdot \max (1, D /(2 \pi)))$, and let $n_{2}$ be given by Lemma 2.7 Let $n_{3} \geq n_{2}$ be so that $\rho^{n_{3}}<1 / 4$.

Let us first deal with the easy case $\sup \left|f^{\prime}\right| \geq 2 C|t| \sup |f|$. Setting $\gamma_{1}=$ $\max \left((2 C|t|)^{-1}, \rho^{n_{3}}+3 / 4\right)<1$, we have $\sup \left|\widetilde{L}_{s}^{n_{3}} f\right| \leq \sup |f| \leq \frac{1}{2 C|t|} \sup \left|f^{\prime}\right| \leq$ $\gamma_{1}\|f\|_{1, t}$, and, by Lemma 2.2 ,

$$
\begin{aligned}
\frac{\left|\left(\widetilde{L}_{s}^{n_{3}} f\right)^{\prime}\right|}{|t|} & \leq C(\Sigma, \bar{K}) \frac{|s|}{|t|} \sup |f|+\frac{\rho^{n_{3}}}{|t|} \sup \left|f^{\prime}\right| \\
& \leq\left(\frac{\sqrt{\left(\max |\sigma|^{2}+|t|^{2}\right)}}{2|t|}+\rho^{n_{3}}\right) \frac{\sup \left|f^{\prime}\right|}{|t|} \leq \gamma_{1}\|f\|_{1, t} .
\end{aligned}
$$

If $\sup \left|g^{\prime}\right|<2 C|t| \sup |g|$, then the function $g^{2}$ satisfies (2.4) for $2 C \max (1, \sup |g|)$ for which Lemmas $2.7,2.8$ hold. Note also that a slight modification of the CauchySchwartz argument in the beginning of the proof of Lemma 2.8 yields

$$
\left|\widetilde{L}_{\sigma}^{m n_{3}}(g)(x)\right|^{2} \leq K \frac{\lambda_{2 \sigma}^{m n_{3}}}{\lambda_{\sigma}^{2 m n_{3}}} \widetilde{L}_{0}^{m n_{3}}\left|g^{2}\right|(x),
$$


for some $K$ independent of $m n_{3}$ and $f$. Next, assume sup $\left|f^{\prime}\right|<2 C|t| \sup |f|$ and assume $\|f\|_{1, t}=1$. By the spectral properties of $\widetilde{L}_{0}$ on the space of Lipschitz functions endowed with the norm $\sup |g|+\operatorname{Lip}(g)$ (with $\operatorname{Lip}(g)$ the smallest Lipschitz constant of $g$ ), there are $R_{\sigma}<\infty, \tau_{\sigma}^{L}<1$ (independent of $f$ and $t$ ), with:

$$
\begin{gathered}
\sup \left|\widetilde{L}_{s}^{2 m n_{3}}(f)\right|^{2} \leq \sup \left|\widetilde{L}_{\sigma}^{m n_{3}}\left(\widetilde{L}_{s}^{m n_{3}}(f)\right)\right|^{2} \leq K \frac{\lambda_{2 \sigma}^{m n_{3}}}{\lambda_{\sigma}^{2 m n_{3}}} \sup \widetilde{L}_{0}^{m n_{3}}\left(\left|\widetilde{L}_{s}^{m n_{3}}(f)\right|^{2}\right) \\
\leq K \frac{\lambda_{2 \sigma}^{m n_{3}}}{\lambda_{\sigma}^{2 m n_{3}}}\left(\int\left|\widetilde{L}_{s}^{m n_{3}}(f)\right|^{2} d \nu_{0}+R_{\sigma}\left(\tau_{\sigma}^{L}\right)^{m n_{3}}[\sup +\operatorname{Lip}]\left(\left|\widetilde{L}_{s}^{2 m n_{3}}(f)\right|^{2}\right)\right) \\
\leq K \frac{\lambda_{2 \sigma-1}^{m n_{3}}}{\lambda_{\sigma}^{2 m n_{3}}} \cdot\left(\sup |f|^{2} \beta^{m}+R_{\sigma}\left(\tau_{\sigma}^{L}\right)^{m n_{3}}[\sup +\operatorname{Lip}]\left(\left|\widetilde{L}_{s}^{m n_{3}}(f)\right|^{2}\right)\right)
\end{gathered}
$$

using Lemma 2.8 for $n=n_{3}$ and Cauchy-Schwartz). Lemma 2.7 gives

$$
\sup \left|\widetilde{L}_{s}^{m n_{3}}(f)\right|^{2}=\sup |f|^{2}\left|v_{m}\right|^{2} \leq \sup |f|^{2} u_{m}^{2} \leq \sup |f|^{2} \leq 1,
$$

and $\operatorname{Lip}\left(\widetilde{L}_{s}^{m n_{3}}(f)^{2}\right) \leq 2 \sup |f|^{2} \cdot \sup |f| \sup \left|v_{m}^{\prime}\right| \leq 2 \sup |f|^{3} 2 C|t| \leq 4 C|t|$, since $\operatorname{Lip}\left(\left|v_{m}\right|\right) \leq \operatorname{Lip}\left(v_{m}\right)=\sup \left|v_{m}^{\prime}\right|$.

In order to find $\max \left(\beta, \tau_{\sigma}^{n_{3}}\right) \cdot \frac{\lambda_{2 \sigma-1}^{n_{3}}}{\lambda_{\sigma}^{n_{3}}}<\gamma_{2}^{2}<1$ so that (for all $m$ )

$$
K \frac{\lambda_{2 \sigma-1}^{m n_{3}}}{\lambda_{\sigma}^{2 m n_{3}}} \cdot\left(\beta^{m}+R_{\sigma} \tau_{\sigma}^{m n_{3}}(1+4 C|t|)\right) \leq \gamma_{2}^{2 m},
$$

it is enough to require $m \geq \widehat{A} \log |t|$ for some $\widehat{A}>0$ (and $\sigma$ close enough to 0 ).

To control the derivative, invoke Lemma 2.2, exploiting the bounds just obtained:

$$
\begin{aligned}
\frac{\sup \left|\left(\widetilde{L}_{s}^{2 m n_{3}}(f)\right)^{\prime}\right|}{|t|} & \leq \frac{C(\Sigma, \bar{K})|s|}{|t|} \sup \left(\widetilde{L}_{\sigma}^{2 m n_{3}}|f|\right)+\frac{\rho^{m n_{3}}}{|t|} \sup \left(\widetilde{L}_{\sigma}^{2 m n_{3}}\left|(f)^{\prime}\right|\right) \\
& \leq \frac{C(\Sigma, \bar{K})|s|}{|t|} \gamma_{2}^{m}+2 C \rho^{m n_{3}} \sqrt{K} \frac{\lambda_{2 \sigma-1}^{m n_{3}}}{\lambda_{\sigma}^{m n_{3}}} \leq \gamma_{3}^{m} .
\end{aligned}
$$

Take $n_{4}=2 n_{3}$ and large enough $A \geq \widehat{A}$.

\section{REFERENCES}

1. N. Anantharaman, Travaux de Dolgopyat sur le mélange des mesures de Gibbs, Chapter 1 of "Géodésiques fermées d'une surface sous contraintes homologiques," unpublished, 2000.

2. V. Baladi and B. Vallée, Euclidean algorithms are Gaussian, Preprint (2003), www.arxiv.org.

3. D. Dolgopyat, On decay of correlations in Anosov flows, Ann. of Math., 147, 1998, pp. 357390. MR1626749 (99g:58073)

4. D. Dolgopyat, Private communication (2003).

5. M. Pollicott, On the mixing of Axiom A attracting flows and a conjecture of Ruelle, Ergodic Theory Dynam. Systems, 19, 1999, pp. 535-548. MR1685406 (2001d:37038)

6. M. Pollicott and R. Sharp, Exponential error terms for growth functions on negatively curved surfaces, Amer. J. Math., 120, 1998, pp. 1019-1042. MR.1646052 (99h:58148)

7. L. Stoyanov, Spectrum of the Ruelle operator and exponential decay of correlations $f$ or open billiard flows, Amer. J. Math., 123, 2001, pp. 715-759. MR1844576 (2002f:37061)

8. L.-S. Young, Statistical properties of dynamical systems with some hyperbolicity, Ann. of Math., 147, 1998, pp. 585-650. MR1637655 (99h:58140)

CNRS, UMR 7586, Institut de Mathématiques de Jussieu, 75251 Paris, France

E-mail address: baladi@math.jussieu.fr

CNRS, GReyC, Université de Caen, 14032 Caen, France

E-mail address: brigitte.vallee@info.unicaen.fr 